\title{
A Grid Based Weather Radar Data Retrieval and Processing Framework*
}

\author{
Diego Arias, John Sanabria, Wilson Rivera \\ Department of Electrical and Computer Engineering \\ University of Puerto Rico at Mayagüez \\ Mayagüez, Puerto Rico 00681
}

\begin{abstract}
In this paper, we describe the implementation of a distributed data retrieval and processing strategy enabled by using grid computing technologies and applied to distributed collaborative adaptive sensing environments. Underlying the grid computing and storage infrastructure there is a data dispersion algorithm to guarantee pervasive data management. Experimental results show that the proposed framework integrates successfully radar networks to grid infrastructures, while providing higher resources utilization than typical storage strategies.
\end{abstract}

Index Terms - Distributed radar network, grid computing, data retrieval and processing.

\section{INTRODUCTION}

The National Science Foundation Engineering Research Center for Collaborative Adaptive Sensing of the Atmosphere (CASA) is focused on developing Distributed Collaborative Adaptive Sensing (DCAS) [1] as a systems technology to improve our ability to monitor the earth's lower atmosphere. DCAS aims to radically alter the radar paradigm. Rather than relying on single radar to provide long range (hundreds of kilometers) coverage, DCAS proposes to mosaic the output of lower power shorter range (tens of kilometers) radars.

The island of Puerto Rico presents a unique set of challenges that accentuate the limitation of the current large radar paradigm. With a land area of approximately $9000 \mathrm{~km}^{2}$, Puerto Rico is home to an estimated 3.8 million people, predominately located in the costal lowlands surrounding a central mountain range peaking $1.3 \mathrm{~km}$ above sea level. The island is located in the Tropic of Cancer and receives a 30year average of 63 inches of rainfall per year. The amount and nature of this rainfall combined with the island's particular orography makes Puerto Rico prone to flash flooding events, which require Quantitative Precipitation Estimation (QPE) for accurate radar based forecasting. QPE forecasts are produced by the NWS forecast office in San Juan using, in part, the island's single NEXRAD WSR-88D, TJUA.

TJUA is located in Cayey on the eastern end of the central mountain range. The radar is installed at an elevation of 851 $\mathrm{m}$, and is responsible for Puerto Rico and the U.S. Virgin Islands. Due to NWS regulations the lowest elevation angle a NEXRAD will use for sampling is $0.5^{\circ}$. As range is increased away from the instrument, so is the minimum altitude that the NEXRAD will sample. The University of Puerto Rico, Mayagüez (UPRM) campus, is located west of TJUA at a distance of approximately $100 \mathrm{~km}$. Over the course of this 100 $\mathrm{km}$ the NEXRAD beam rises $871 \mathrm{~m}$ into the atmosphere.
When combined with the base elevation of TJUA, the resulting minimum altitude of atmosphere being sampled is $1.7 \mathrm{~km}$ above sea level.

Sampling the atmosphere nearly $2 \mathrm{~km}$ above sea level can only provide an estimate of the ground truth rainfall. Precipitation may be blown by wind or evaporate before falling to the ground which results in unavoidable errors in rainfall estimation introduced by the location where sampling takes place. Correctly estimating ground truth rainfall requires sampling the atmosphere close to the earth's surface making the mission of QPE ideally suited to the DCAS paradigm of remote sensing. Utilizing radars with a maximum range of no more than 30 kilometers would, assuming an elevation angle of $0.5^{\circ}$, result in a minimum altitude of $261 \mathrm{~m}$ at maximum range. In addition $30 \mathrm{~km}$ radars could be located in the costal low lands on tall buildings roof tops allowing the beam minimum altitude to remain bellow $300 \mathrm{~m}$. A total of $430 \mathrm{~km}$ radars, properly located, would be required to provide complete coverage of the island. Varying the range of radars within a sensing network would allow the network to adapt to the requirements of the terrain, allowing radar's beam altitude to be kept bellow $1 \mathrm{~km}$, impacting QPE measurements and the corresponding flood forecasts. To verify this hypothesis a QPE test-bed is being constructed on Puerto Rico. The infrastructure and technologies derived from this QPE test-bed will be used to build the base for the Puerto Rico Full DCAS System Testbed.

Clearly, the fully realization of the DCAS paradigm requires efficient data retrieval and processing algorithms. Accordingly, the Parallel and Distributed Computing Laboratory (PDCLab) at UPRM is collaborating with CASA team to facilitate a distributed computing and storage infrastructure. The PDClab Grid Testbed, deployed at the University of Puerto Rico-Mayaguez, is an experimental grid designed to address research issues such as the effective integration of sensor and radar networks to grid infrastructures. In this paper, we describe the deployed infrastructure and discuss further the underlying data retrieval and processing strategy.

The remainder of this paper is organized as follows. In Section II, we describe the grid enabled tested at UPRM including computing infrastructure and the Student Testbed (STB) grid portal. The distributed data retrieval and processing method is described in Section III. In Section IV, we present experimental results. Related work is discussed in section V. Finally, we conclude this paper and provide our future plan in Section VI.

\footnotetext{
* This material is based upon work supported by the National Science Foundation under Grants No. 0313747 and No. 0424546.

Any opinions, findings, and conclusions or recommendations expressed in this material are those of the author(s) and do not necessarily reflect the views of the National Science Foundation
} 


\section{DATA GRID INFRASTRUCTURE}

The availability of powerful computers and high-speed network technologies as low-cost commodity components has changed the way we solve large scale problems. These technology opportunities have led to the possibility of using geographically distributed computers as a single, unified computing resource. Grid computing [2] enables coordination, storage and networking of resources across geographically dispersed organizations in a transparent way for users. The first generation of grid technologies has demonstrated the feasibility of grids for addressing challenging large scale problems (e.g. GridPhyN and Teragrid among many others).

\section{A. The PDCLab Grid Tested}

The PDClab Grid Testbed is an experimental grid designed to address research issues such as the effective integration of sensor and radar networks to grid infrastructures. The PDClab grid test-bed components run CentOS 4.2 and the Globus Toolkit 4.0.1. The computational resources available on the grid include an IBM xSeries Linux cluster with 64 nodes, dual processor at $1.2 \mathrm{GHz}, 53 \mathrm{~GB}$ of memory and $1 \mathrm{~TB}$ of storage; Eight (8) IA-64 Itanium servers, dual processor at $900 \mathrm{MHz}$, each with 8GB of memory and 140GB of SCSI Ultra 320 storage; Two (2) IA-32 Pentium IV servers, dual processor at $3 \mathrm{GHz}$, each with 1GB of memory and 120GB of ATA-100 storage; One (1) IA-32 Pentium III server, dual processor at $1.2 \mathrm{GHz}$ with 2GB of memory and 140Gb of SCSI Ultra 160 storage; One (1) IA-32 Xeon server, dual processor at 2.8 $\mathrm{GHz}$, L2 Cache 1MB with 1GB of memory and one 230 GB RAID of storage (STB Server); and two (2) PowerVault storage with 8TB. Figure 1 shows a schematic of the GridService based infrastructure.

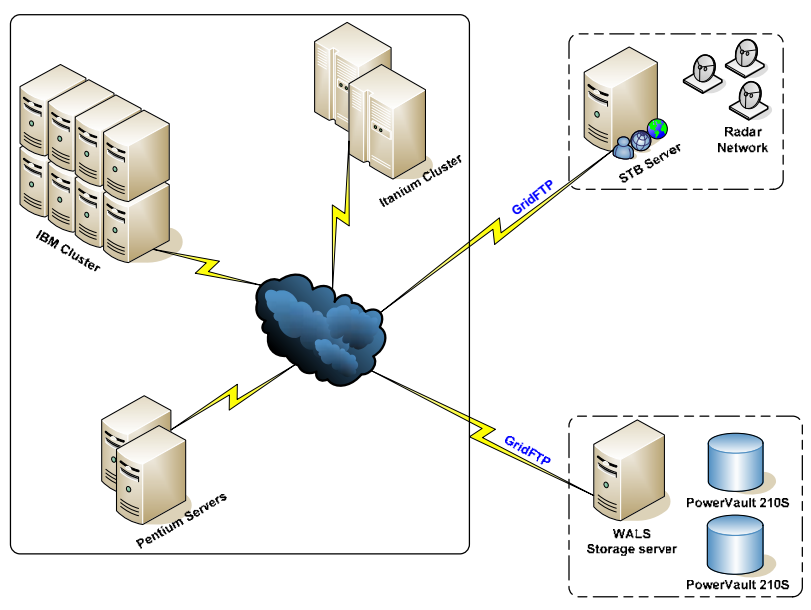

Figure 1: PDCLab Grid Testbed

\section{B. The STB Grid Portal}

The Student Testbed (STB) is being led by a multi-level, multi-institution team of students, ranging from a variety of scientific and engineering fields located at the core CASA partner campuses. The testbed focuses on constructing a network of radars to provide detailed QPE in a tropical environment, such as Puerto Rico, while educating students and advancing radar technology. The STB serves as an educational experience tool to students by allowing them to install a sit-and-spin X-band magnetron radar on top of the Electrical and Computer Engineering building at UPRM. This particular radar is a single-polarization radar with limitations in the control of the azimuth and elevation scanning, yet it will have the capabilities to detect rain and help characterize rain attenuation at X-band.

To establish the required infrastructure for the distributing component of the system we have considered applying grid computing technologies and exploring the ability to manipulate and/or storage data from different radars, execute algorithms on different platforms in a transparent way to end users, and ultimately provide a functional platform for students' experimentation.

Grid portals are becoming very popular platforms to provide friendly and simplified interfaces to grid services and resources such as applications, data servers, application servers, clusters, sensing instruments, and so on. Grid portals based on a web portal model, offer external developers personalization and customization features through modular, extendable and reusable software components which may be developed independently from the purpose or the portal architecture. These modules (called portlets) are Java technology based web components, managed by a portal container, which act as an intelligent dispatcher attending process requests and forwarding the proper response to the client through dynamic content. In addition portlets provide a presentation layer for Information (or Grid) Systems. The GridSphere portal framework [3] is a portlet JSR (Java Standard Resource) compliant container that allows portlet development through a set of classes and tools for web applications. Furthermore, this framework has included a collection of core services and portlets, providing easy environment to grid portals development.

The Grid Portal interface ensures a transparent mechanism for accessing resources and grid services. Gridsphere is the selected framework to develop the STB Grid portal. The developed portlets provide a presentation layer for the manipulation of both, processed data and raw-data from radar, and for the services for end-users. Additionally, portlets make the visualization of weather information, such as reflectivity in order to estimate rainfall rate over the western area of Puerto Rico. Figure 2 is a snapshot of the grid portal interface.

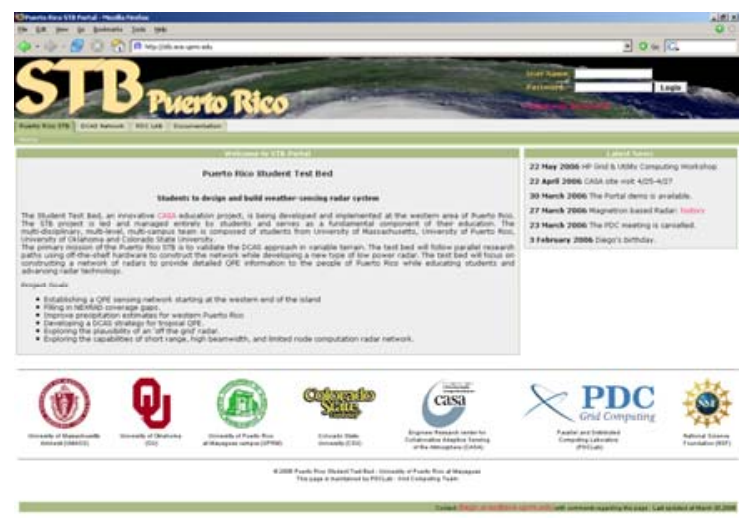

Figure 2: STB Grid portal interface 
The grid portal provides current rainfall estimates over the western area of Puerto Rico through reflectivity displays. This information is unrestricted and is available for anyone who accesses the portal. Figure 3 shows how the base reflectivity information corresponding to a sweep is plotted over the Mayaguez area.

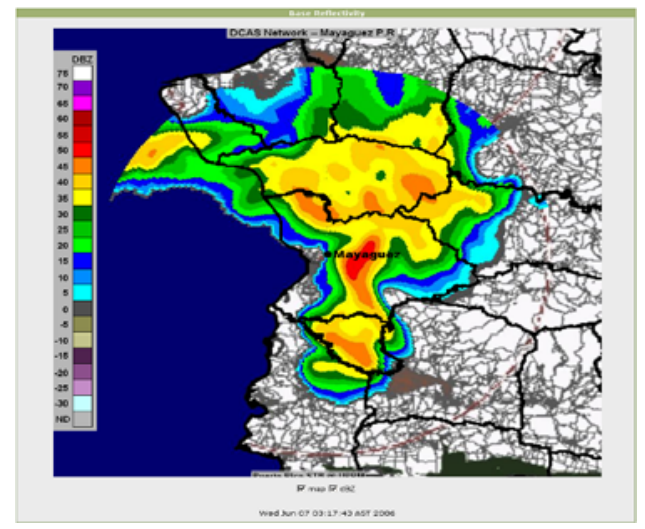

Figure 3: Base Reflectivity Portlet.

\section{DAta Retrieval AND PRocessing}

In our framework we propose to deploy a set of low price storage servers integrated to the sensor network. Sensor nodes collect and transmit data to local storage units. Data files are then partitioned and blocks are replicated to other storage units. When blocks have the independent probability of failure $p$, the access reliability is determined by:

$$
p(a)=\sum_{i=0}^{n-m}\left(\begin{array}{l}
n \\
i
\end{array}\right) p^{i}(1-p)^{n-i}
$$

Let $F=b_{1}, b_{2}, b_{3}, \ldots$ be a file, where $b_{i}$ is an integer taken from a certain range $[0 \ldots(2 B-1)]$. If $b_{i}$ is two bytes long, as an example, then $0 \leq b_{i} \leq 65535$. Let $c$ be a prime number greater than $b_{i}$. Each $b_{i}$ is an element of the finite field $Z_{c}$ where all arithmetic operations are done in $\bmod c$. Since $c>$ $(2 B-1)$, this implies an excess of one bit per byte when integers greater than $(2 B-1)$ are obtained, which implies a storage space increment. In order to avoid the waste of space, all $b_{i}$ values are represented as polynomials with binary coefficients $\left(b_{B} x^{B}+b_{(B-1)} x^{(B-1)}+\ldots+b_{1} x+b_{0}\right)$ and use a larger degree non-reducible polynomial $p(x)$ instead the prime $p$. The polynomial must suffice $\left(p(x) \in Z_{2}[x]\right)$ in such a way that all operations can be done in the finite Galois field $E=G F(2 B)$.

An Information Dispersal Algorithm (IDA) based replication strategy [4] has been deployed as a composite service on top of the Globus toolkit following the WSRF specification. The components of the IDA service include a service to select the adequate places to store the file pieces (adapter service) and a service to split and recover files (IDA based service). Each service is an independent functional entity so it can be re-used on other service deployment. In our deployment GridFTP [5] is used to transfer files among resources, and GSI (Globus Security Infrastructure) [6] services are used to certificate management and authentication.

GridFTP is a secure, high-performance and robust data transfer mechanism used to access remote data. In addition to GridFTP, Globus provides Globus Replica Catalog to maintain a catalog of dataset replicas so that, instead of duplicating large datasets, only necessary pieces of the datasets are stored on local hosts. The Globus Replica Management software provides the replica management capabilities for data grid by integrating the replica catalog and GridFTP.

The Grid Security Infrastructure is used by the Globus Toolkit for authentication and secure communication. GSI is implemented using public key encryption, X.509 certificates, and the secure sockets layer (SSL) communication protocol and incorporates single sign-on and delegation.

GKrellM $^{1}$, a third party monitor tool, is used to monitor and collect data related to memory and disk space in resources. The adapter service takes into consideration information related to both disk space and GridFTP service availability on specific resources to determine the number of file chunks and places to store pieces of files. The IDA-based service then implements the partition and store of the pieces of files.

The following steps describe how data distribution is performed.

1. Execute the IDA (dispersal mode) over each raw-data file stored in the server, in such a way that $\boldsymbol{n}$ chunks are obtained.

2. Check if the required credential to access the grid is valid. If it has expired, then the renewal will be necessary. Select $\boldsymbol{n}$ nodes from all the available nodes on the grid.

3. Send the chunks to the grid using a 1:1 distribution, using gsiftp as the transport protocol. Use the globusurl-copy command

4. Register in separate log files all the information related to the dispersed files, such as path, name, size and IDA settings.

5. Delete the original raw-data file.

\section{A CASE STUDY}

Experiments involving time measurements vs. data size, take as reference data size from National Climatic Data Center. This data is a Level II base data, available in compressed tape archive format. It contains data per day from specific NEXRAD Level II radar. The compressed data for a day is about $150 \mathrm{MB}$, while uncompressed is about 2.3GB. Note that this is the data mount for 24 hours of continuous scan. For rain fall measurements and precipitation estimation, the primary implementation of DCAS network requires less than 8 hours of continuous scan. Considering all the exposed before and the limited transmission due to wireless communication as mentioned, testing is achieved with data size range from $100 \mathrm{MB}$ to $1 \mathrm{~GB}$.

\footnotetext{
${ }^{1}$ http://members.dslextreme.com/users/billw/gkrellm/gkrellm.html
} 
Figure 4 shows the resources used when NCtoJPG process is executed in the server and when it is executed in the grid. It is shown that the local execution is faster (3 times more) than remote execution if we are processing a single file. The multijob test was performed with 6 files which imply 6 different jobs. Execution over the grid of the multi-job was achieved using GRAM [7], meanwhile in the local execution, each job ran sequentially. Upon executing the experiments, only four nodes were available, including the STB server. The total time used in a local submission is half the total time used in a distributed execution.
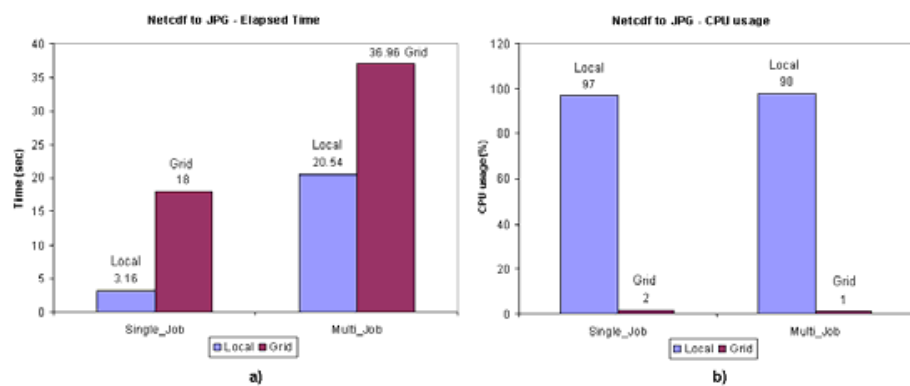

Figure 4: Resources utilization.

In terms of process time, a multi-job executed in the server shows a better response than a multi-job executed over the grid. However, a local-job involves more resources usage than remote-jobs.

Preliminary experimentation have been also performed using a grid enabled virtualized environment. The configuration of each virtual node uses space in disk of 6 GB and $256 \mathrm{MB}$ of RAM. These virtual nodes are deployed on physical servers running Fedora Core 6 and Xen as virtualization platform. We have also used CentOS as operating system and VMWare as virtualization platform with similar results to those presented in this paper. For the experiments in this paper, we use four physical servers. A server running the client application, other server acting as gateway, and other two servers hosting one virtual node and four virtual nodes, respectively.

The experimental results for our case study show that utilization of resources of the gateway and virtual organization implementations is not significant compared to the application and transport components themselves. Figure 5 shows the dispersion computational cost for sets of data files of 50MB, 150MB, and 300MB, respectively.

\section{RELATED WORK}

One of the major today's research challenges is the development of adaptive systems for analyzing and predicting the atmospheric conditions. Weather conditions often occur suddenly and evolve rapidly in such a way that an adaptive observation is necessary to perform accurate weather forecasting. Several research projects are focused on achieving this goal, in fact, multidisciplinary teams across multiple institutions are involved in this process. The Linked Environments for Atmospheric Discovery (LEAD) [8], is a framework for identifying, accessing, assimilating, forecasting,
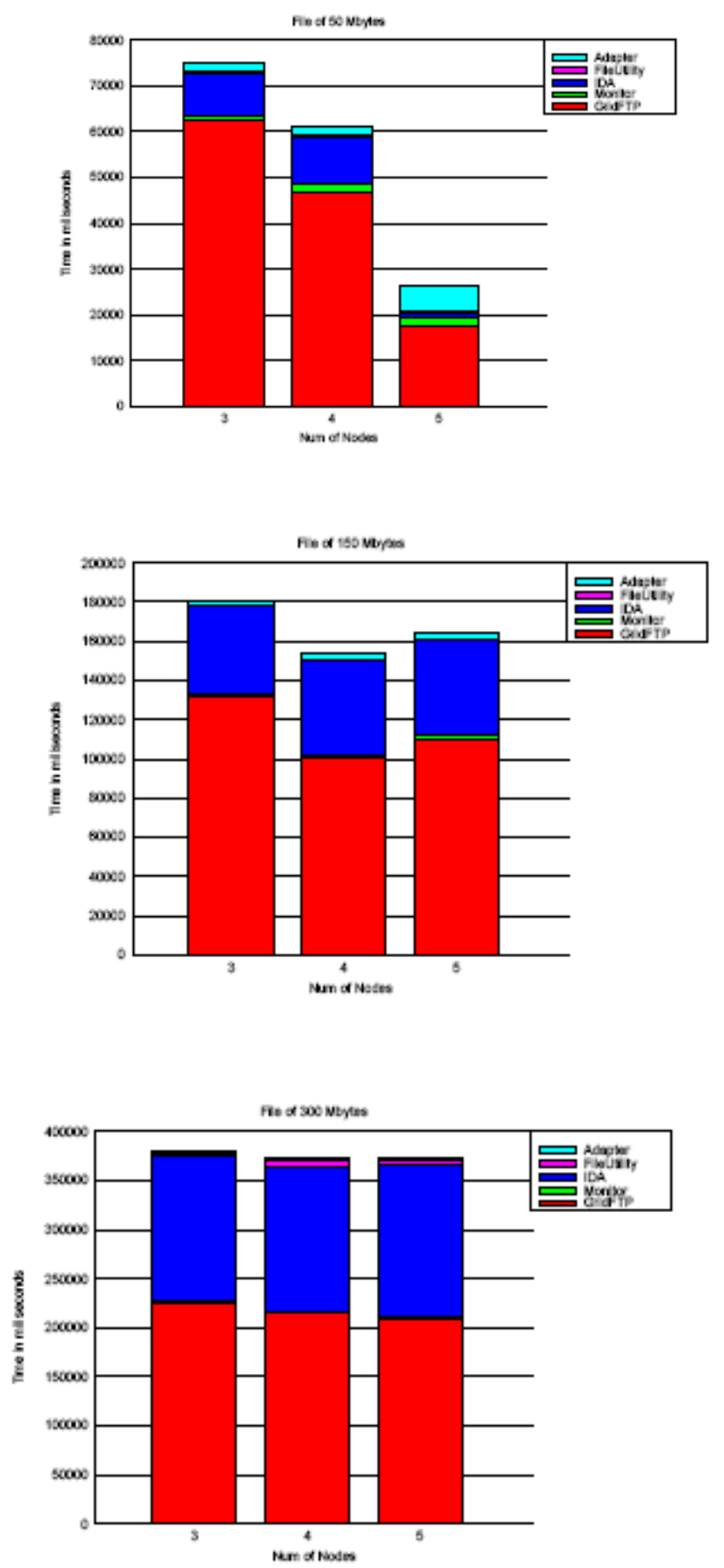

Figure 5: Dispersion on a Grid enabled virtualization

managing, analyzing, mining and visualizing a broad array of meteorological data and model output independent of format and physical location. The LEAD system is dynamically adaptable in terms of time, space, forecasting and processing.

The LEAD system can be catalogued as a Service Oriented Architecture (SOA). SOAs are widely deployed in the commercial enterprise sector and they are the foundation for many scientific grid technologies. The LEAD SOA includes technologies and tools, such as, Globus toolkit, Unidata's Local Manager (LDM), Open-Source Project for a Network 
Data Access Protocol (OpenDap) and the OGSA Data Access and Integration (OGSA-DAI) service.

The LEAD portal is based on the NSF National Middleware Initiative Open Grid Computing Environment Portal toolkit (OGCE). This portal allows users authentication through proxy identity certificates, based on the Globus GSI model. Once user identity is verified, the LEAD grid, its services and resources will be made available for its users.

The work in this paper is complementary to the LEAD project as we propose a new information dispersion strategy and demonstrate the viability of its implementation on a platform that combines grid and virtualization technologies.

In a Service Oriented Architecture (SOA) environment, resources on a network are made available as independent services that can be accessed without knowledge of their underlying platform implementation. SOA is not tied to a specific technology and may be implemented using a wide range of interoperability standards including Remote Procedure Call (RPC) and Web Services. In a SOA environment, provisioning refers to the creation, maintenance and deactivation of services, while orchestration describes the automated arrangement, coordination, and management of such services.

$\mathrm{Xu}$ [9] proposes service migration in distributed virtual machines as a method to implement adaptive infrastructures. To facilitate managing heterogeneous resources, the virtual machine technology provides a powerful layer of abstraction and allows multiple applications to multiplex the resources in a distributed system. The main target in this work is to achieve service reconfiguration in face of server overload or failures. The service migration mechanism allows moving computational services of a virtual server, for instance a shared array runtime support system, to available servers for adaptive grid computing. Thus, jobs can resume computation on a remote server without requiring service pre-installation. A better understanding of the impact of virtualization technologies on the service provisioning and orchestration would e helpful to attain adaptive infrastructure running under uncertainty.

\section{CONCLUSIONS}

In this paper, we have presented a dispersion algorithm for weather radar data and its implementation on a grid based infrastructure powered with virtualization technologies. Experimental results obtained in a case study for dispersion/replication of data files demonstrate the viability of the proposed environment. From the experimental results it is not clear how different number of provisioned resources impact the application performance. We truly believe a further analysis of different provisioning and orchestration protocols will contribute to a better understanding of resources interaction and the inherent service and resource uncertainty.

\section{REFERENCES}

[1] McLaughlin, D., J. Brotzge, V. Chandresakar, K. Droegemeier, J. Kurose,
B. Philips, M. Preston, and S. Sekelsky, 2004: Distributed Collaborative Adaptive Sensing for Hazardous Weather Detection, Tracking, and Predicting. International Conference on Computational Science 2004.

[2] Foster, C. Kesselman, J. Nick, and S. Tuecke (2002), "The physiology of the Grid: An open Grid services architecture for distributed systems integration, Technical report, Open Grid Service Infrastructure WG, Global Grid Forum.

[3] D. Gannon, G. Fox, M. Pierce, B. Plale, G. von Laszewski, C. Severance, J. Hardin, J. Alameda, M. Thomas, J. Boisseau, Grid Portals: A Scientist's Access Point for Grid Services, 2003

[4] D. Arias and W. Rivera , Using Grid Computing to Enable Distributed Radar Data Retrieval and Processing. IEEE International Conference on Parallel and Distributed Computing, Applications and Technologies, 2006.

[5] W. Allcock, J. Bresnahan, R. Kettimuthu, M. Link, C. Dumitrescu, I. Raicu, I. Foster. The Globus Striped GridFTP Framework and Server Proceedings of Super Computing 2005 (SC05), November 2005.

[6] I. Foster, C. Kesselman, G. Tsudik, S. Tuecke. A Security Architecture for Computational Grids Proc. 5th ACM Conference on Computer and Communications Security Conference, pp. 83-92, 1998.

[7] J. Nabrzyski, J.M. Schopf, J. Weglarz (Eds). Grid Resource Management Kluwer Publishing, Fall 2003.

[8] Droegemeier K.K.; Gannon D.; Reed D.; Plale B.; Alameda J.; Baltzer T.; Brewster K.; Clark R.; Domenico B.; Graves S.; Joseph E.; Murray D.; Ramachandran R.; Ramamurthy M.; Ramakrishnan L.; Rushing J.A.; Weber D.;Wilhelmson R.; Wilson A.; Xue M.; Yalda S. Service-oriented environmentsfor dynamically interacting with mesoscale weather. Computing in Science \&Engineering, 7(6):12\{29, Nov.-Dec. 2005.

[9] C.-Z. Xu, "Service migration in distributed virtual machines for adaptive grid computing," in ICPP '05: Proceedings of the 2005 International Conference on Parallel Processing (ICPP'05). Washington, DC, USA: IEEE Computer Society, 2005, pp. 358-365. 